\title{
Association between Chronic Physical Illness and Internalizing Symptomatology in a Transdiagnostic Clinical Sample of Youth
}

Elizabeth Wolock, BA ${ }^{1,2}$, Alexander Queen, $\mathrm{PhD}^{2}$, Gabriela M. Rodríguez, PhD, HSPP ${ }^{3}$, John R. Weisz, PhD, ABPP ${ }^{1}$

${ }^{1}$ Harvard University, Department of Psychology, ${ }^{2}$ Tufts University, Department of Psychology, ${ }^{3}$ Indiana University School of Medicine, Department of Psychiatry

\section{Acknowledgements}

We thank our colleagues in the Harvard Laboratory for Youth Mental Health for the contribution of data to this study. This research was made possible via funding from the Connecticut Health and Development Institute to John Weisz. We acknowledge, with thanks, the contributions of Dr. Kerri Modry-Mandell, Dr. Spencer Evans, and Nathan Hollinsaid to the study.

This is the author's manuscript of the article published in final edited form as:

Wolock, E. R., Queen, A. H., Rodríguez, G. M., \& Weisz, J. R. (2020). Chronic Illness and Internalizing Symptomatology in a Transdiagnostic Clinical Sample of Youth. Journal of Pediatric Psychology, 45(6), 633-642. https://doi.org/10.1093/jpepsy/jsaa028 


\begin{abstract}
Objective: In research with community samples, children with chronic physical illnesses have shown elevated anxiety and depressive symptoms, compared to healthy peers. Less is known about whether physical illnesses are associated with elevated internalizing symptoms even among children referred for mental health treatment — a pattern that would indicate distinctive treatment needs among physically ill children receiving mental health care. We investigated the relationship between chronic physical illness and internalizing symptomatology among children enrolling in outpatient mental health treatment.
\end{abstract}

Method: 262 treatment-seeking children ages 7-15 and their caregivers completed a demographic questionnaire, Child Behavior Checklist, and Youth Self-Report during a pretreatment assessment. Physical illnesses were identified through caregiver report.

Results: There was no overall association between presence/absence of chronic physical illness and parent- or child-reported symptoms. However, number of chronic physical illnesses was related to parent- and child-reported affective symptoms. Children with two or more chronic physical illnesses had more severe depressive symptoms than those with fewer physical illnesses. Conclusion: Having multiple chronic illnesses may elevate children's risk of depression symptomatology, even in comparison to other children seeking mental health care. This suggests a need to identify factors that may exacerbate depression symptoms in physically ill children who are initiating therapy, and to determine whether different or more intensive services may be helpful for this group. The findings suggest the potential utility of screening for depression in youth with chronic physical illnesses, as well as addressing mental and physical health concerns during treatment. 


\section{Association between Chronic Physical Illness and Internalizing Symptomatology in a Transdiagnostic Clinical Sample of Youth}

Approximately $26.6 \%$ of children in the United States suffer from at least one chronic illness (Van Cleave, Gortmaker, \& Perrin, 2010). Chronic illness refers to illness that "lasts three months or more, affects a child's normal activities, and requires frequent hospitalizations, home health care, and/or extensive medical care" (Mokkink, van der Lee, Grootenhuis, Offringa, \& Heyans, 2008, p. 1442). Chronic illnesses have three primary features - they are prolonged in their duration, do not resolve spontaneously, and are rarely cured completely (Stanton, Revenson, \& Tennen, 2007). While many chronic illnesses common to children, such as asthma, diabetes, and skin-related conditions, have no known cure, they can typically be managed medically. To manage the physical illnesses, children and their families have to attend frequent medical appointments, maintain daily medical regimens, and accommodate various physical changes (Perrin, 1999; Van Cleave et al., 2010).

The difficulty of managing chronic illnesses and related limitations on social and physical activities are two factors associated with the well-established comorbidity between physical and mental illness in youth. Research has shown that symptoms of mental disorders are up to four times as prevalent in children with chronic illness compared to children who are physically well (Hysing, Elgen, Gillberg, Lie, \& Lundervold, 2007; Pinquart \& Shen, 2011b). Across the spectrum of psychopathology, there is a particularly strong association between physical illness and internalizing symptomatology (Bennett, 1994; Butler et al., 2018; Last, Stam, Onland-van Nieuwenhuizen, \& Grootenhuis, 2008; Pao \& Bosk, 2010; Pinquart \& Shen, 2011b), particularly anxiety and depression symptoms. 
Several studies have examined the relationship between physical illness and internalizing symptomatology. With regard to anxiety, previous studies have found a positive correlation between chronic illnesses and anxiety symptoms (Bakula et al., 2019; Butler et al., 2018; Chavira, Garland, Daley, \& Hough, 2009; LeBlanc, Goldsmith, \& Patel, 2003; Pao \& Bosk, 2010, Pinquart \& Shen, 2011a). When further investigating this relationship, LeBlanc et al. (2003) found that children are at highest risk for developing anxiety within the first year of diagnosis, likely due to adjustment to the diagnosis and care regimen (Drotar, 2006). Furthermore, factors such as illness intrusiveness and illness-related stigma have been found to contribute to anxiety symptoms in youth with chronic illness (Bakula et al., 2019). Researchers have also examined depressive symptoms in children with chronic medical problems (Ferro, Gorter, \& Boyle, 2015; Pinquart \& Shen, 2011b; Bennett, 1994). In a meta-analysis, Pinquart and Shen (2011) found increased depressive symptoms in youth with chronic physical illness relative to youth without chronic illness, with the largest effect sizes among females. Based on previous research demonstrating that female adolescents are more likely than males to respond to stressors with depressive symptoms (Piccinelli \& Wilkinson, 2000), the authors hypothesized that there may be a stronger effect of chronic illness on depressive symptoms in females.

In addition to examining the mental health outcomes of youth with chronic illnesses, it is important to consider the effects of experiencing multiple chronic illnesses on youth mental health. While there has been little research done examining the effects of multiple chronic illnesses on internalizing symptoms, Newacheck and Stoddard (1994) performed a study examining the prevalence and impact of experiencing multiple childhood chronic illnesses. The National Health Interview Survey on Child Health surveyed roughly 17,000 children, and found that $5 \%$ of children had two or more physical illnesses, while less than $1 \%$ had three or more 
physical illnesses. Results showed that children with multiple chronic illnesses had significantly more emotional and behavioral problems than both children with one and zero physical illnesses. Furthermore, while not specifically examining internalizing symptoms, Reynolds, Boergers, Kopel, and Koinis-Mitchell (2018) found that, in a sample of children with asthma, having comorbid medical conditions not only exacerbated asthma symptoms but also negatively impacted academic outcomes and sleep.

This supports previous findings linking poorly managed chronic illness to higher severity of mental illness. Specifically, the results indicate that there may be a cumulative effect of managing multiple chronic illnesses - the more physical illnesses a child has, the more difficult it is to manage them. However, it is important to acknowledge that Newacheck and Stoddard (1994) did not report a breakdown of "emotional and behavioral problems" (e.g. anxiety, depression, and conduct), which is necessary to fully understand which specific mental illness symptoms are most affected. Understanding this would allow researchers and clinicians to focus their treatment efforts on specific areas, thus providing the most effective treatment for children.

Most of the existing literature has examined the relationship between physical and mental illness in samples of children recruited for having a chronic illness. However, few studies have taken the opposite approach and examined medical comorbidity in samples of children with mental illnesses (Chavira et al., 2009; Sawyer, Whaites, Rey, Graetz, \& Baghurst, 2002). Chavira et al. (2009) specifically examined the impact of medical comorbidity on mental and functional health outcomes among youth recruited for having at least one anxiety disorder. Approximately half of the sample also had a comorbid physical illness. Allergies and asthma were the most common comorbid physical illnesses in children with anxiety, and those with comorbid physical illnesses exhibited higher levels of emotional problems than those without 
physical illnesses. Sawyer et al. (2002) also examined physical health in samples of children with mental illnesses, but used Health-Related Quality of Life (HRQL), defined as a "multifactorial construct that focuses on individuals' perceptions of their physical, psychological, and social functioning" (p. 532) as the primary measurement. This research found a higher percentage of physical health problems in children with mental disorders, which included Major Depressive Disorder (MDD), Conduct Disorder (CD), and Attention-Deficit/Hyperactivity Disorder (AD/HD). The research team also found that children with MDD were the most likely to have physical health problems.

Existing literature shows that children who are physically ill are significantly more likely to have emotional and behavioral symptoms. However, only a few studies have examined whether children with diagnosed emotional and behavioral disorders are at higher risk for having comorbid physical illnesses. Furthermore, there is very little research examining the association between number of physical illnesses and internalizing symptoms, which may assist in identifying youth in particular need of treatment. The current study examined the relationship between internalizing symptoms and chronic physical illness in a transdiagnostic sample of children seeking outpatient mental health care. Based on the aforementioned research showing a strong correlation between chronic physical illness and internalizing symptoms, it was hypothesized that children with a chronic physical illness would have higher self-reported and parent-reported anxiety and depressive symptoms, as well as a positive relationship between number of chronic physical illnesses and internalizing symptoms.

\section{Method}

\section{Participants}


Participants were referred by clinicians at one of four community mental health clinics in Connecticut. Out of 683 participants referred, 262 children and adolescents completed the baseline assessment. The sample was $48.47 \%$ female $(n=127)$. Children ranged in age from 7 to 15 years, $M=10.79, S D=2.48$. Participants were primarily Non-Hispanic White, Black, and Latino/Hispanic, and more than half of the sample (64.12\%) had an annual combined income of less than $\$ 40,000$. Further demographic information can be found in Table 1 .

Inclusion criteria were: (1) endorsement during phone screens by caregiver of the child having problems related to anxiety, depression, conduct, or posttraumatic stress and (2) borderline (T-score $\geq 65$ ) or clinical elevations (T-score $\geq 70$ ) on the Internalizing and/or Externalizing subscales of the Child Behavior Check List (CBCL) and/or the Youth Self Report (YSR) at the baseline assessment. Exclusion criteria were: (1) court-ordered clinical services, (2) a diagnosis of intellectual disability, schizophrenia, autism spectrum disorder, or psychotic symptoms, (3) hospitalization in past year for suicide attempt or intent, or (4) absence from Connecticut for more than six weeks for the six months following baseline assessment.

\section{Measures}

Demographic characteristics and protocol assignment. During the baseline assessment, primary caregivers provided demographic information about youth participants, including age, sex, racial/ethnic background, and medical history, via interview. Caregivers were also asked to report whether or not their child had any physical illness (i.e. "Does your child have any significant physical illnesses/medical problems? If yes, please list them here.”). The number of physical illnesses listed was used to classify participants into groups of zero, one, or two or more illnesses. All participants with physical illnesses had exclusively chronic illnesses, except for two participants who had bronchitis and Lyme disease. As both bronchitis and Lyme 
disease can transform from acute to chronic illnesses, this study is focused on chronic illnesses. There were three children with three illnesses listed, and they were grouped with those that had two for analytic purposes. Participants were classified as having or not having asthma based on whether caregivers included asthma in the list of physical illnesses/medical problems in response to the above question. Finally, information about the treatment protocol each child was assigned to at the start of treatment (i.e., anxiety, depression, trauma, or conduct) was obtained from study assessment records.

Child Behavior Checklist (CBCL/6-18; Achenbach \& Rescorla, 2001). The CBCL is a 112-item, caregiver-report questionnaire that assesses behavioral and emotional problems in youth. The present study used the T-scores of the following subscales: Internalizing problems, Affective problems, and Anxiety problems. The CBCL was administered to all caregivers at baseline. Internal consistency for the CBCL is typically between 0.75 and 0.84 , as measured by Cronbach's alpha (Nakamura, Ebesutani, Bernstein, \& Chorpita, 2009). Internal consistency was very high for the current overall sample, Cronbach's $\alpha=0.99$. Internal consistency was also high for the individual subscales used in analyses: Cronbach's alphas between 0.93 and 0.96 for the three subscales included.

Youth Self-Report (YSR; Achenbach \& Rescorla, 2001). The YSR is a 112-item, selfreported questionnaire based on the CBCL. The scales used for this study were the same as those used for the CBCL. The YSR was administered to all participants in the study at baseline. Question \#96 ("I think about sex too much") was deemed inappropriate for participants under the age of 11 and thus was removed in those cases. The present study used the T-scores of the following subscales: Internalizing problems, Affective problems, and Anxiety problems. Internal consistency was very high for this sample, Cronbach's $\alpha=0.99$. Internal consistency was also 
high for the individual subscales used in analyses: Cronbach's alphas between 0.98 and 0.99 for the three subscales included.

\section{Procedure}

The current study is a secondary analysis of baseline data from a randomized controlled trial comparing the effects of the Modular Approach to Therapy for Children with Anxiety, Depression, Trauma, or Conduct Problems (MATCH-ADTC; Chorpita \& Weisz, 2009) under two different approaches to implementation support for clinicians. Clinic staff were asked to refer any child between ages 7 and 15 seeking treatment at their site to study staff for screening. Families who agreed and provided their contact information were contacted to complete a phone screen, and if eligible, to schedule a baseline assessment. Phone screens and baseline assessments were administered by Bachelor's and Master's level research assistants.

Caregivers and youth participants completed their respective portions separately, but with the same assessor. The caregiver portion included a demographic questionnaire and the CBCL while the child portion only included the YSR. After completion of the baseline assessment, participants and caregivers were compensated for their time. The study was approved by the Internal Review Board (IRB) of Harvard University as well as the IRB of the Department of Children and Families of the state of Connecticut.

\section{Planned Analyses}

Prior to analysis, continuous variables were examined for normality. All continuous variables were found to be normal, indicated by skewness and kurtosis values of less than an absolute value of two. Descriptive analyses were used for demographic data as well as the demographic profiles of different illnesses. Independent sample t-tests were used for comparing CBCL and YSR scales between children with and without a physical illness. Additional 
independent sample t-tests were performed for youth with asthma, due to the very high number of participants in the sample with asthma $(n=52)$. Analysis of Variance (ANOVA) and Tukey HSD/Dunnett's C post-hoc tests were used to examine the relationship between number of illnesses and CBCL and YSR subscale scores. The sample had a missing data rate of less than $1.5 \%$ of the total data, and participants with missing data were excluded from analyses.

\section{Results}

\section{Sample Characteristics}

Sample demographics and descriptive statistics are presented in Tables 1 and 2. Mean CBCL Internalizing symptoms $(M=64.21, S D=9.59)$ were in the borderline clinical range, and Affective symptoms $(M=64.96, S D=8.96)$ and Anxiety symptoms $(M=62.54, S D=8.58)$ were below the borderline clinical cutoff. Mean YSR Internalizing symptoms $(M=56.61, S D=$ 11.79), Affective symptoms $(M=59.29, S D=9.16)$, and Anxiety symptoms $(M=58.11, S D=$ 7.86) T-scores were all below the borderline clinical cut-off of 60 .

For youth with chronic illness, the most commonly endorsed parent-rated affective symptoms were feeling depressed and poor sleep, and the most common youth-rated symptoms were crying and poor eating. In the Anxiety symptoms subscale, the most commonly endorsed parent-rated symptoms were relying on adults, feeling nervous and feeling worried, and the most common youth-rated symptoms were general fears and feeling worried. Further information on individual item endorsement can be found in Table 3. Primary problem areas (Anxiety, Depression, Trauma, or Conduct), according to the MATCH-ADTC protocol, as well as physical illness presence as measured via parent report are presented in Table 2. The Depression (40.0\%) and Conduct (38.1\%) protocols were the most common primary problem areas, followed by Anxiety (19.5\%) and Trauma (2.4\%). 


\section{Chronic Physical Illness Presence and Internalizing Symptoms}

Our first hypothesis predicted that children with a chronic physical illness would have higher parent-reported internalizing symptoms, as measured by the Internalizing problems, Affective problems, and Anxiety problems subscales of the CBCL. Means and standard deviations for these variables can be found in Table 4. An independent samples $t$-test revealed no significant difference in the mean CBCL Internalizing subscale T-scores between children who had a chronic illness and those who did not, $t(101.55)=-0.23, p=.83$. There were also no significant differences in the CBCL Affective, $t(255)=-1.48, p=.14$, or Anxiety subscale $\mathrm{t}$ scores, $t(255)=-0.18, p=.86$, between children who did and did not have a chronic illness. When examining only asthma and internalizing symptoms, there were no significant differences in the CBCL Internalizing, $t(255)=-0.051, p=.96$, Affective, $t(255)=-1.03, p=.230$, or Anxiety, $t(255)=-0.38, p=.71$ subscales between children who did and did not have asthma.

We also hypothesized that children with a chronic physical illness would have higher mean child-rated internalizing symptoms compared to children without a chronic physical illness. Means and standard deviations for child-rated measures can be found in Table 4. An independent samples $t$-test revealed no significant difference in the YSR Internalizing subscale tscores between children who did and did not have a chronic illness, $t(241)=1.47, p=.14$. Furthermore, no significant differences were found between children with and without chronic physical illnesses on the YSR Affective, $t(241)=0.89, p=.37$, and Anxiety subscales, $t(241)=$ $1.73, p=.08$. When examining only asthma and internalizing symptoms, there were no significant differences in the YSR Internalizing, $t(241)=2.07, p=.07$, Affective, $t(258)=-0.36$, $p=.72$, or Anxiety, $t(241)=1.24, p=.22$, subscales between children with and without asthma.

\section{Number of Chronic Illnesses and Internalizing Symptoms}


The second hypothesis examined more specifically the relationship between number of chronic physical illnesses $(0,1$, or $2+)$ and parent- and child-reported internalizing symptoms. Means and standard deviations can be found in Table 4. A one-way ANOVA showed that the number of chronic illnesses a child had was not significantly associated with CBCL Internalizing subscale t-scores, $F(2,256)=2.580, p=.078$, or Anxiety subscale t-scores, $F(2,256)=0.527, p$ $=.591$. There was, however, a significant mean difference between groups on Affective subscale t-scores, $F(2,256)=5.098, p=.007, \eta^{2}=0.038$. Levene's test revealed a lack of homogeneity of variances for the Affective T-scores, so Dunnett's C post hoc analysis was used. Post hoc analyses indicated that children with two or more illnesses had significantly higher CBCL Affective T-scores than both children with one chronic illness and children with no chronic illnesses, $p$ 's $<.05$. There was no significant difference between children with one chronic illness and children with no illnesses.

A one-way ANOVA revealed a significant effect of number of physical illnesses a child had $(0,1$, or $2+)$ on YSR Internalizing subscales T-scores, $F(2,242)=3.044, p=.049, \eta^{2}=$ 0.025. However, post hoc analyses failed to detect any significant differences between the three groups (p-values ranged from .089 to .43). A significant effect of number of illnesses was also found on YSR Affective subscale t-scores, $F(2,242)=6.46, p=.002, \eta^{2}=0.051$. A Tukey HSD post hoc test revealed that children with two or more chronic illnesses had higher YSR Affective t-scores than children with one chronic illness $(p=.002)$ and children with no chronic illnesses $(p=.028)$. There was no significant difference between children with one chronic illness and children with no illness, $p=.077$. An ANOVA showed that the number of chronic illnesses a child had was not significantly associated with YSR Anxiety subscale t-scores, $F(2,242)=$ $1.381, p=.253$ 


\section{Exploratory Analyses}

To explore the clinical relevance of the previous finding, chi-square analyses were utilized to examine potential differences in the proportion of youth with clinically elevated affective symptoms (those with a CBCL or YSR T-score of 70 or higher) based on the absence or presence of chronic illness. A significantly higher percentage of youth with chronic illnesses had clinically elevated parent-reported affective symptoms compared to youth without chronic illnesses, $\chi^{2}(1, \mathrm{~N}=257)=7.26, p=.007$. However, there was no significant difference in youthreported clinically elevated affective symptoms between youth with chronic illness and youth without chronic illness $\chi^{2}(1, \mathrm{~N}=243)=.179, p=.067$.

\section{Discussion}

This study examined the relationship between chronic physical illness and internalizing disorders among a transdiagnostic sample of youth referred for mental health treatment. We hypothesized that children with a chronic physical illness would experience more internalizing symptoms than children without a chronic physical illness. Furthermore, we hypothesized that children with a greater number of chronic illnesses would report more severe internalizing symptoms. Our hypotheses were partially supported. The overall presence of a chronic illness was not associated with higher youth- and caregiver-rated internalizing symptoms. However, the number of chronic illnesses was associated with both the CBCL and YSR Affective subscale scores. Specifically, children with two or more chronic illnesses reported significantly more severe affective symptoms than those with one chronic illness and those with no chronic illnesses. There was no significant difference between the affective symptoms of children with one chronic illness and those with no chronic illnesses. Taken together, these findings suggest 
that having two or more chronic illnesses places youth at increased risk for more severe affective symptoms. This relationship was not found for the Total Internalizing and Anxiety subscales.

\section{Chronic Physical Illness Presence and Internalizing Symptoms}

The lack of a significant relationship between the presence of pediatric chronic illness and overall internalizing symptoms is contrary to most findings in the research literature, as prior research has found that children with chronic illnesses are more likely than children without chronic illnesses to have various emotional and behavioral difficulties (Butler et al., 2018; Last et al., 2006; Pao \& Bosk, 2010; Pinquart \& Shen, 2011). There are a few possible explanations for the discrepancy between this finding and the extant literature. First, our sample consisted of youth who were referred for treatment of emotional and behavioral disorders rather than chronic illness. As a result, there were no exclusion criteria related to presence or type of chronic illness, which led to a wide variety of chronic illnesses. In most previous research, the variety of physical illnesses in the sample has been controlled for or limited to one illness (Pao \& Bosk, 2010; Pinquart \& Shen, 2011). Second, the chronic illnesses affecting the youth in our sample were generally low in severity. For example, the majority of youth with a chronic illness in our sample $(n=52)$ were reported to have asthma, which is often low in severity and well-managed (Letitre, Groot, Draaisma, \& Brand, 2014). This is further supported by the non-significant findings when we compared only those children who did and did not have asthma. Thus, it is possible that the relatively low overall severity of chronic illnesses in our sample may have limited the ability to detect an effect of presence of chronic illness on internalizing symptoms.

\section{Number of Illnesses and Internalizing Symptoms}

As both the parent and child reports indicated, children with two or more illnesses had significantly more affective symptoms than those with one or no chronic illnesses. This may be 
the case because of the difficulty that comes with managing multiple physical illnesses.

Managing multiple chronic illnesses can entail taking multiple medications and frequent doctors' visits, both of which can be burdens for children and families. Furthermore, when children have more than one chronic illness, they can be limited in what activities they can engage in, both socially and physically, which could create a sense of social withdrawal as well as low selfesteem (Bennett, 1994; Ferro \& Boyle, 2015). This, in turn, may lead to feelings of depression (Kovacs \& Devlin, 1998). In addition, children with severe, chronic physical illnesses often feel a loss of control, which can lead to feeling hopeless with regard to their future (Shaw \& McCabe, 2007). Furthermore, work by Newacheck and Stoddard (1994) on the prevalence and impact of multiple childhood chronic illnesses found that children with multiple chronic conditions had more emotional and behavioral problems than other children, which is reflected in our findings of higher depressive symptoms in youth with multiple chronic illnesses. Last, according to work by Sawyer et al. (2002), there was a strong relationship between youth MDD and chronic health problems. Since the Affective Problems subscale is meant to capture symptoms of Persistent Depressive Disorder (PDD) and MDD, it appears that, in our sample, this relationship also holds true.

Overall, this finding is consistent with the existing literature, such as the study done by Ferro et al. (2015), which examined trajectories of depressive symptoms among youth with chronic illnesses. Ferro et al. found that youth with chronic illnesses not only had more severe depressive symptoms than their healthy peers, but also experienced high depressive symptoms during early- and mid-adolescence, which reflects the age range of a large portion of the sample in the current study. This finding is reflected by the exploratory analyses, which revealed that a higher proportion of youth with chronic illnesses had clinically elevated parent-reported 
depressive symptomatology. However, this difference was not found among youth report, which is consistent with previous studies in which clinically-referred youth reported lower internalizing symptomatology than their caregivers (Weisz et al., 2019).

Despite findings indicating that having more chronic illnesses was associated with increased depressive symptoms, there was no relationship between number of illnesses and anxiety symptoms or overall internalizing symptoms. While we do not have data on age of onset of chronic illness in our sample, it is possible that youth in our study had been living with their chronic illnesses for years. We speculate that this may hold especially true among children who have more than one illness, because many of the illnesses were genetic or autoimmune disorders with early ages of onset. According to LeBlanc et al. (2003), children are at very high risk for development of behavioral and emotional problems as they adjust to a chronic illness diagnosis. Specifically, children with chronic illnesses are most likely to develop anxiety within the first year of diagnosis (LeBlanc et al., 2003, p. 862). Therefore, it is possible that children in the present study had more anxiety at the onset of the chronic illness, but have since gained a better understanding, have fewer unknowns, and therefore feel less anxious.

Based on the findings of no relationship between number of illnesses and anxiety symptoms, we can also understand why the Internalizing subscale did not reveal a significant relationship. On both the YSR and CBCL, the Internalizing subscale includes all items from the Somatic Complaints, Anxiety, and Affective subscales (Nakamura et al., 2009). Therefore, the results were likely skewed by the lack of significance for anxiety symptoms. In addition, it is important to note that $40.0 \%$ of the sample had a primary problem area of depression. Anxiety was only the primary problem area of $19.5 \%$ of the sample. Therefore, having twice as many children with depression as anxiety, could have led to a non-significant relationship between 
number of illnesses and total internalizing symptoms, which includes both depression and anxiety.

\section{Clinical Implications}

One clinical implication of these findings is the importance of screening for depression in youth who have chronic physical illnesses. This holds especially true in children who are suffering from more than one chronic illness. In hospitals or other medical settings, children often have more attention paid towards their physical health than their mental health (Wissow, van Ginneken, Chandna, \& Rahman, 2016). Coping with chronic physical illnesses is difficult, and thus mental health screenings and preventative measures are needed (Foy, Kelleher, \& Laraque, 2010).

In addition, mental health providers in community outpatient youth mental health clinics, such as the ones in this study, should continue to take time to understand the background and medical history of a child. This includes inquiring about physical health at intake assessments and throughout treatment and assessing how children are managing their chronic conditions throughout the course of treatment, as applicable. For children with comorbid physical illnesses, clinicians must consider how treatment may need to be altered to be most appropriate and effective. Specifically, the content of treatment may need to be tailored to address illness-related issues associated with affective symptoms. As one example, Szigethy et al. (2014) have created and tested a cognitive-behavioral therapy (CBT) protocol specifically for youth who experience depression in combination with inflammatory bowel disease.

Another option could entail incorporating mental and physical health treatment into one integrated protocol, to help children and families better manage having multiple illnesses. This type of treatment could be implemented in primary care settings, both during children's yearly 
visits and visits for chronic illnesses. For example, psycho-educational group intervention for children with chronic illnesses, such as the one developed by Last et al. (2007), could be implemented in primary care clinics. Last et al. used components of CBT, such as relaxation, cognitive restructuring, and general psycho-education, for children with chronic illnesses in hospital settings. They found that their intervention greatly reduced internalizing symptoms and improved quality of life. In addition, Weersing, Gonzalez, Campo, and Lucas (2008) showed that implementing brief behavioral therapy (including relaxation and problem-solving strategies) in primary care settings for youth with anxiety and/or depression was very effective in reducing internalizing symptoms. Similar interventions could be very useful not only in helping youth manage their physical illnesses but also in reducing their depressive (and anxious, if applicable) symptoms. A more holistic understanding of all the pertinent factors would likely lead to more efficient treatment of mental health issues, especially depression, in children who have comorbid chronic illnesses.

\section{Limitations}

This study was a secondary cross-sectional analysis, using baseline assessment data from a large-scale community treatment study. Therefore, the primary study was not designed specifically for the present analyses. One limitation was the way in which the data on chronic illnesses were collected on the demographic questionnaire, given that examining chronic illness was not a primary focus of the original study. Parents were first asked if their child had any physical illnesses (without a definition of "physical illness" provided) and were given space to list three. This did not allow parents the opportunity to list if their child had more than three illnesses or any descriptions of the listed illnesses. In addition, the physical illnesses were parentreported, which is less reliable than physician reports. Furthermore, while parents were asked to 
list their child's allergies, there was no standardized question on type and severity of allergies, which prevented us from examining the relationship between allergies and internalizing symptoms.

The age range of the participants was fairly wide and youth were not evenly spread across age groups, which prevented us from examining youth age as a moderator in our analyses. Missing data in the current study was also a limitation, though less than $1.5 \%$ of the total data for each subscale analyzed was missing. This typically occurred when children were not able to or chose not to complete the assessment, even though their parents had. The YSR scores of the children with missing data were excluded from the YSR analyses. Last, while some of the findings were statistically significant, the effect sizes were small, suggesting caution in interpreting and generalizing the findings.

Finally, the limiting sample characteristics must be acknowledged. First, there was a large imbalance in the number of children who had and did not have physical illnesses. While the data followed a normal distribution, the imbalance in number of children who had and did not have physical illnesses affected the analyses and limited the generalizability of the results. This led to a lack of linearity and large amount of variance in some of the CBCL and YSR scales, especially the Anxiety subscales. Furthermore, over $75 \%$ of the participants in this study were middle- or lower-income families (household income of 0-59,000 dollars per year). While this demographic is unique from most of the existing research, and thus a strength, it also signifies a lack of socioeconomic diversity in the sample population. Regardless of the overall low socioeconomic status of the families, they were still able to receive mental health services through insurance, which is not available to all children who may need such services.

\section{Conclusion and Future Directions}


Chronic physical illness is a risk factor for emotional and behavioral issues in children. The present study suggests specifically that having more than one chronic illness may be a further risk factor for internalizing problems, especially depression, even in a mental health treatment-seeking transdiagnostic sample. Given these conclusions and the aforementioned limitations, future research is warranted in this area. Research examining the nature and severity of chronic illnesses would help us better understand some of the unexpected results of the present study. To do so, a more comprehensive questionnaire that clearly defines physical illnesses and includes physician verification of physical illness would be needed. Gathering information on age of onset and severity of physical illness, as well as a complete list of physical illnesses would help researchers to better understand possible moderating factors. It would also be important to gather information on what treatments children are receiving for their chronic illness, how many and what specialty (if any) of physicians children are seeing, and whether a child has received previous mental health treatment. Analyses of this information, in tandem with socioeconomic status data, may enhance our understanding of how access to treatment relates to mental health outcomes.

Furthermore, research examining age as a moderator of the relationship between chronic physical illness and internalizing symptoms is warranted. This would allow researchers to understand how developmental stage affects coping with mental and chronic illnesses. Previous research (Cousino \& Hazen, 2013; Nabors et al., 2013) has also found that factors such as parenting stress, external support, and effective communication are often related to psychosocial outcomes in chronically ill youth, so examination of these potential moderators is warranted. In addition, further research examining the various mediators of the relationship between chronic illness and affective symptoms is warranted. Potential mediators to test are reduction in quality 
of life and emotion dysregulation. Last, a longitudinal study design is warranted to better test and understand the directionality of the association between mental and physical health, and how comorbid chronic illness may affect treatment outcomes. 


\section{References}

Achenbach, T. M, \& Rescorla, L. A. (2001). The manual for the ASEBA school-age forms \& profiles. Burlington, VT: University of Vermont, Research Center for Children, Youth, and Families.

Angeli, E., Wagner, J., Lawrick, E., Moore, K., Anderson, M., Soderlund, L., \& Brizee, A. (2010, May 5). General format. Retrieved from http://owl.english.purdue.edu/owl/resource/560/01.

Bakula, D., Sharkey, C., Perez, M., Espeleta, H., Hawkins, M., Chaney, J., \& Mullins, L. (2019). The role of stigma in the relationship between illness intrusiveness and adjustment in adolescents and young adults: A path model. Journal of Pediatric Psychology, 44(5), 611-619.

Butler, A., Van Lieshout, R., Lipman, E., MacMillan, H., Gonzalez, A., Gorter, J.,...Ferro, M. (2018). Mental disorder in children with physical conditions: A pilot study. BMJ Open, 8, $1-10$.

Bennett, D. (1994). Depression among children with chronic medical problems: A meta-analysis. Journal of Pediatric Psychology, 19(2), 149-169.

Centers for Disease Control and Prevention. (2013). Mental health surveillance among children United States, 2005-2011. Morbidity and mortality weekly report, 62(2), 1-35.

Chavira, D., Garland, A., Daley, S., \& Hough, R. (2008). The impact of medical comorbidity on mental health and functional outcomes among children with anxiety disorders. Journal of Developmental and Behavioral Pediatrics, 29(5), 394-402.

Chorpita, B., \& Weisz, J.R. (2009). Modular approach to therapy for children (MATCH) with anxiety, depression, trauma, or conduct problems. PracticeWise. 
Cousino, M., \& Hazen, R. (2013). Parenting stress among caregivers of children with chronic illness: A systematic review. Journal of Pediatric Psychology, 38(8), 809-828.

Drotar, D. (2006). Psychological interventions in childhood chronic illness. Washington, D.C.: American Psychological Association.

Ferro, M., \& Boyle, M. (2015). The impact of chronic physical illness, maternal depressive symptoms, family functioning, and self-esteem on symptoms of anxiety and depression in children. Journal of Abnormal Child Psychology, 43(1), 177-187.

Ferro, M., Gorter, J., \& Boyle, M. (2015). Trajectories of depressive symptoms during the transition to young adulthood: The role of chronic illness. Journal of Affective Disorders, 174, 594-601.

Foy, J.M., Kelleher, K.J., Laraque, D. (2010). Enhancing pediatric mental health care: Strategies for preparing a primary care practice. American Academy of Pediatrics, 125(3), 87-108.

Hysing, M., Elgen, I., Gillberg, C., Lie, S.A., \& Lundervold, A.J. (2007). Chronic physical illness and mental health in children. Results from a large-scale population study. Journal of Child Psychology and Psychiatry, 48(8), 785-792.

Kovacs, M., \& Devlin, B. (1998). Internalizing disorders in childhood. The Journal of Child Psychology and Psychiatric and Allied Disciplines, 39(1), 47-63.

Last, B., Stam, H., Onland-van Nieuwenhuizen, A., \& Grootenhuis, M. (2008). Positive effects of a psycho-educational group intervention for children with a chronic disease: first results. Patient Education and Counseling, 65(1), 101-112.

LeBlanc, L., Goldsmith, T., \& Patel, D. (2003). Behavioral aspects of chronic illness in children and adolescents. The Pediatric Clinics of North America, 50, 859-878. 
Letitre, S., Groot, E., Draaisma, E., \& Brand, P. (2014). Anxiety, depression and self-esteem in children with well-controlled asthma: case-control study. Archives of Disease in Childhood, 99(8), 744-748.

Mokkink, L., van der Lee, J., Grootenhuis, M., Offringa, M., \& Heyans, H. (2008). Defining chronic diseases and health conditions in childhood (ages 0-18 years of age): National consensus in the Netherlands. European Journal of Pediatrics, 167(12), 1441-1447.

Nabors, L., Kichler, J., Brassell, A., Thakker, S., Bartz, J., Pangollo, J., ... Lundy, H. (2013). Factors related to caregiver state anxiety and coping with a child's chronic illness. Family, Systems, and Health, 31(2), 171-180.

Nakamura, B., Ebesutani, C., Bernstein, A., \& Chorpita, B. (2009). A psychometric analysis of the child behavior checklist DSM-oriented scales. Journal of Psychopathology and Behavioral Assessment, 31(3), 178-189.

National Institute of Mental Health. (2017). Health and education: statistics. Retrieved from https://www.nimh.nih.gov/health/statistics/index.shtml.

Newacheck, P., \& Stoddard, J. (1994). Prevalence and impact of multiple childhood chronic illnesses. The Journal of Pediatrics, 123(10), 40-48.

Pao, M., \& Bosk, A. (2010). Anxiety in medically ill children/adolescents. Depression and Anxiety, 28(10), 40-49.

Perrin, J. (1999). Chronic illness. Journal of Developmental and Behavioral Pediatrics, 3, 335.

Piccinelli, M., \& Wilkinson, G. (2000). Gender differences in depression. British Journal of Psychiatry, 177, 486-492.

Pinquart, M., \& Shen, Y. (2011a). Anxiety in children and adolescents with chroic physical illness: a meta-analysis. Acta Paediatrica, 100(8), 1069-1076. 
Pinquart, M., \& Shen, Y. (2011b). Depressive symptoms in children and adolescents with chronic physical illness: An updated meta-analysis. Journal of Pediatric Psychology, 36(4), 375-384.

Reynolds, K., Boergers, J., Kopel, S., \& Koinis-Mitchell, D. (2018). Multiple comorbid conditions, sleep quality and duration, and academic performance in urban children with asthma. Journal of Pediatric Psychology, 43(9), 943-954.

Szigethy, E., Bujoreanu, S. I., Youk, A. O., Weisz, J. R., Benhayon, D., Fairclough, D., .. . DeMaso, D.R. (2014). Randomized efficacy trial of two psychotherapies for depression in youth with inflammatory bowel disease. American Academy of Child and Adolescent Psychiatry, 53(7), 726-735.

Sawyer, M.G., Whaites, L., Rey, J.M., Graetz, B.W., \& Baghurst, P. (2002). Health-related quality of life of children and adolescents with mental disorders. Journal of the American Academy of Child and Adolescent Psychiatry, 41(5), 530-537.

Shaw, S., \& McCabe, P. (2008). Hospital-to-school transition for children with chronic illness: Meeting the new challenges of an evolving health care system. Psychology in the Schools, 45(1), 74-87.

Stanton, A.L., Revenson, T., Tennen, H. (2007). Health psychology: Psychological adjustment to chronic disease. Annual Review of Psychology, 58, 565-592.

Van Cleave, J., Gortmaker, S.L., \& Perrin, J.M. (2010). Dynamics of obesity and chronic health conditions among children and youth. The Journal of the American Medical Association, 303(7), 623-630. 
Weersing, R., Gonzalez, A., Campo, J., \& Lucas, A. (2008). Brief behavioral therapy for pediatric anxiety and depression: piloting an integrated treatment approach. Cognitive and Behavioral Practice, 15(2), 126-139.

Weisz, J. R., Bearman, S. K., Ugueto, A. M., Herren, J. A., Evans, S. C., Cheron, D. M., ... Jensen-Doss, A. (2019). Testing robustness of Child STEPs effects with children and adolescents: A randomized controlled effectiveness trial. Journal of Clinical and Adolescent Psychology, 1-13.

Wissow, L.S., van Ginneken, N., Chandna, J., \& Rahman, A. (2016). Integrating children's mental health into primary care. Pediatric Clinics of North America, 63(1), 97-113. 